\title{
Water and Salt Stress Metabolomics for Wheat Genotypes of India
}

\author{
G. KAUR and B. AsTHIR* \\ Department of Biochemistry, Punjab Agricultural University, Ludhiana-141004, Punjab, India \\ (Received 5 January 2018; Accepted 10 July 2019; \\ Communicated by A. Goyal)
}

\begin{abstract}
Shoots of ten day old seedlings of nineteen wheat genotypes were evaluated for proline metabolism, $\mathrm{H}_{2} \mathrm{O}_{2}$, thiobarbituric acid reactive substances (TBARS) and 2,2 diphenyl-picrylhydrazyl (DPPH) radical scavenging activity under water deficit, water withholding and salinity stress conditions. Principle component analysis demarcated four groups: i.e. drought tolerant (Excalibar, Krichauff, Babax, Drysdale, Gladius and C306), salt tolerant (Kharchia, Type11, Krl 1-4 and Krl 19), low stress tolerant (C273, C518 and C591) and susceptible (HD2967, PBW621, WH1105, HD3086, PBW660 and PBW175). Salt stress treatment affected the length, fresh weight and dry weight of seedlings of all studied genotypes in comparison to water deficit and water withholding condition which may be due to higher contents of TBARS. Shoots of salt and drought tolerant genotypes possessed higher proline content and DPPH radical scavenging activity alongwith reduced content of TBARS in parallel with decreased $\mathrm{H}_{2} \mathrm{O}_{2}$ content under water stress conditions. The activities of proline synthesizing enzymes i.e. pyrroline-5-carboxylate synthetase (P5CS) and pyrroline-5-carboxylate reductase (P5CR) were significantly higher than proline degrading enzyme viz. proline dehydrogenase $(\mathrm{PDH})$ under water stress as compared to salinity stress conditions. Overall, results indicated that P5CS, P5CR and PDH activities led to higher build up of proline under water stress, which might play a significant role in improving membrane stability by increasing radical scavenging activity and finally imparting stress tolerance in specific wheat genotypes.
\end{abstract}

Keywords: abiotic stress, drought, salinity, proline, wheat

\section{Introduction}

Drought and salinity are major environmental stresses that cause osmotic stress, reduction in plant growth and crop productivity (Hussain and Mumtaz 2014). Around 45\% of the world agricultural lands are subject to continuous or frequent drought, and worldwide area mapped as being affected by salinity is more than $3 \times 10^{6} \mathrm{~km}^{2}$ i.e. $6 \%$ of the total land area (Lobell et al. 2011). Stress responses in plants causes significant increases of reactive oxygen species in the form of singlet oxygen, superoxide, hydroxyl radical and hydrogen peroxide $\left(\mathrm{H}_{2} \mathrm{O}_{2}\right)$ (Ahmad et al. 2012). It has been visualized that products of lipid peroxidation are formed from polyunsaturated fatty acids that include small hydrocarbon ligands such as ketones, malondialdehyde (MDA) and its related compounds related (Garg and Manchanda 2009). Some of these ligands react with thiobarbituric acid to form col-

*Corresponding author; E-mail: b.asthir@rediffmail.com 
oured compounds called thiobarbituric acid reactive substances (TBARS) (Hameed et al. 2012). By maintaining lower level of $\mathrm{H}_{2} \mathrm{O}_{2}$ content and reduced lipid peroxidation, partial acclimation to stress conditions is generally achieved (Rao et al. 2013).

Plants usually accumulate an array of metabolites including compatible osmolytes particularly amino acids such as proline. The predominant pathway for proline production is its de novo synthesis from glutamate, although decrease in catabolism and enhanced proteolysis is also depicted as an alternate way of synthesis (Kavi Kishore et al. 2005). In plants, proline is mainly synthesized from glutamate via glutamate dehydrogenase (GDH), which is further reduced to glutamate-semialdehyde (GSA) by pyrroline-5-carboxylate synthetase (P5CS) enzyme and spontaneously converts into pyrroline-5-carboxylate (P5C). Thereafter, pyrroline-5-carboxylate reductase (P5CR) reduces the P5C intermediate to proline (Miller et al. 2009). Catabolism of proline occurs in mitochondria via the sequential action of proline dehydrogenase ( $\mathrm{PDH}$ ) which produces $\mathrm{P} 5 \mathrm{C}$ from proline and further converts P5C to glutamate (Szabados and Savouré 2009).

The present study aimed to evaluate the activities of the enzymes involved in proline metabolism alongwith cross functioning of other biochemical molecules and defining complete process of abiotic stress in the shoots of nineteen diversified wheat genotypes. An attempt has been made to gain insight to identify genotypes in responses of proline metabolism and their potential roles under water and salt stress.

\section{Material and Methods}

Nineteen wheat genotypes, representing different agro-climatic zone of Indian wheat cultivation area viz. C306, C273, C591, C518, Type 11, Excalibar, Krichauff, Babax, Gladius, Drysdale, Kharchia, Krl 1-4, Krl 19, PBW 175, HD2967, PBW621, WH1105, HD3086 and PBW660 were the subject of study under salinity and drought stress conditions (Table 1). These genotypes were procured from the Department of Plant Breeding and Genetics, Punjab Agricultural University, Ludhiana (Punjab, India). All the seeds were treated with $0.1 \%$ mercuric chloride for $1 \mathrm{~min}$ and washed thoroughly with sterilized water under aseptic conditions. Seeds were germinated in sterilized petriplates on germination paper imbibed with $5 \mathrm{ml}$ of distilled water at $25^{\circ} \mathrm{C}$ in the dark under control, water deficit, water withholding and salt stress conditions. Water deficit conditions were generated by using 8\% polyethylene glycol (PEG-6000) solution and to generate water withholding conditions, water was with held for two days. Salinity stress conditions were maintained by using $300 \mathrm{mM} \mathrm{NaCl}$ solution. The seedlings were watered with distilled water regularly for three days and at the fourth day with PEG solution in water deficit and $\mathrm{NaCl}$ solution in salt stress whereas water was withheld for two days in water withholding condition (Mahboobet al. 2018).

\section{Growth Parameters}

The shoot lengths were measured at tenth day of post germination (DPG) under control, water deficit, water withholding and salt stress conditions with meter scale. Fresh bio- 


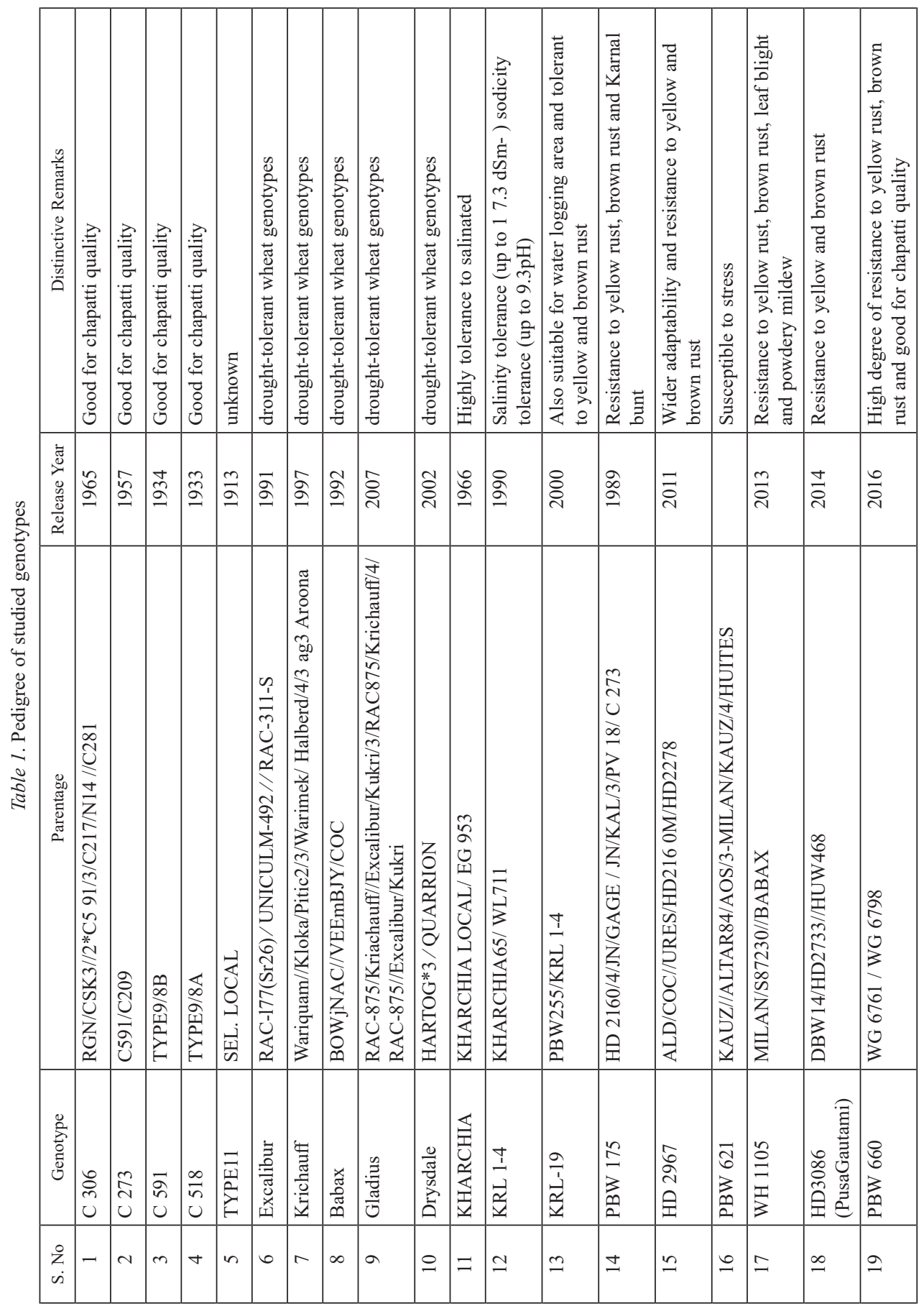


mass of shoots were measured at the same day using weighing balance. For the dry weight analysis, shoots were dried at $60^{\circ} \mathrm{C}$ in oven (12-16 hours) till constant weight (Mahboob et al. 2018).

\section{Extraction and estimation of $\mathrm{H}_{2} \mathrm{O}_{2}$}

$\mathrm{H}_{2} \mathrm{O}_{2}$ was extracted from $500 \mathrm{mg}$ of fresh shoot tissue that was homogenized in $1.5 \mathrm{ml}$ of chilled $0.1 \%$ TCA. The obtained homogenate was filtered usingmuslin cloth and then centrifuged at $10,000 \times \mathrm{g}$ for $15 \mathrm{~min}$ at $4{ }^{\circ} \mathrm{C}$. The supernatant was used for the estimation of $\mathrm{H}_{2} \mathrm{O}_{2}$ content ( $\mathrm{Li}$ et al. 2011).

\section{Extraction and estimation of Malondialdehyde content}

The concentration of malondialdehyde was determined in the terms of thiobarbituric acid reactive substances (TBARS) content (Yu et al. 2015).

\section{Extraction and estimation of proline}

The proline content was estimated by spectrophotometrically (Mwadzingeni et al. 2016).

\section{Extraction and estimation of DPPH radical scavenging activity}

Shoot tissue $(100 \mathrm{mg})$ were homogenized with $2 \mathrm{ml}$ methanol and centrifuged at 10,000 $\mathrm{g}$ for $20 \mathrm{~min} .1 \mathrm{ml}$ supernatant was added to $3 \mathrm{ml}$ ethanol solution of DPPH radical (Sahitya 2018).

\section{Measuring of stress tolerance index (STI) of wheat}

Drought tolerance index was measured by modification of formula as described by Kaur et al. (2014) as follows:

$$
S T I=\frac{(Y s)(Y n)}{\left(Y n^{\sim}\right)^{2}}
$$

Where $Y_{S}-$ DPPH radical scavenging activity/content of $\mathrm{H}_{2} \mathrm{O}_{2}$, TBARS or proline/shoot length of given genotype in stress condition, $Y n-\mathrm{DPPH}$ radical scavenging activity/ content of $\mathrm{H}_{2} \mathrm{O}_{2}$, TBARS or proline/shoot length of given genotype in non-stress condition, $\mathrm{Yn}^{\sim}$ - mean DPPH radical scavenging activity/content of $\mathrm{H}_{2} \mathrm{O}_{2}$, TBARS or proline/ shoot length of given genotype in non-stress condition.

Stress resistance levels were determined by using the median value of STI for each biochemical parameter studied under respective stress condition. The genotypes possessing STI value greater than median value for DPPH radical scavenging activity, proline content, length and biomass of shoot and those with lesser than median value for content of $\mathrm{H}_{2} \mathrm{O}_{2}$ and TBARS were marked positive for stress resistance level. 


\section{Enzymatic assay for proline metabolism}

Shoot tissue (100 mg) was homogenized in prechilled pestle and mortar in the extraction medium containing $100 \mathrm{mM}$ potassium phosphate buffer ( $\mathrm{pH} 7.4), 10 \mathrm{mM}$ 2-mercaptoethanol, $1 \mathrm{mM}$ EDTA, 1\% polyvinylpolypyrrolidone (w/v), $5 \mathrm{mM} \mathrm{MgCl}_{2}$ and $0.6 \mathrm{M} \mathrm{KCl}$. The homogenate was centrifuged at $12,000 \mathrm{~g}$ for $20 \mathrm{~min}$ at $4{ }^{\circ} \mathrm{C}$ and the resulting supernatant was kept at $20^{\circ} \mathrm{C}$ and used for enzymatic assays (Bhaskara et al. 2015).

Glutamate dehydrogenase activity was assayed from the shoots (Abd Elgawadet al. 2015). The enzymatic activity of pyrroline-5-carboxylate synthetase (P5CS) was determined in terms of decrease in NADPH spectrophotometrically at $340 \mathrm{~nm}$ and was ex-

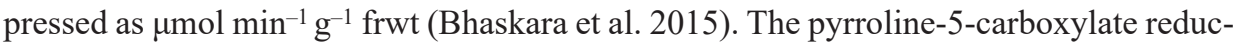
tase (P5CR) activity was determined by (Abd Elgawad et al. 2015). Activity of proline dehydrogenase (PDH) was determined by monitoring the reduction of $\mathrm{NADP}^{+}$at $340 \mathrm{~nm}$ in $0.15 \mathrm{M}$ sodium carbonate buffer ( $\mathrm{pH} 10.3$ ) containing $15 \mathrm{mM}$ l-proline and $1.5 \mathrm{mM}$ $\mathrm{NADP}^{+}$(Bhaskara et al. 2015).

\section{Statistical analysis}

The data was analyzed in a factorial completely randomized design (CRD) by using CPCSI software package (Cheema and Singh 1991). Values are presented as a means $\pm \mathrm{SD}(\mathrm{n}=3)$ and are represented as bars in graph. Multivariate Principle Component Analysis was applied to categorize the genotypes (Kaur et al. 2014).

\section{Results}

\section{Growth and biomass}

Water deficit, water withholding and salt stress treatments caused a significant decrease in the shoot length and biomass of C273, HD2967, PBW621, WH1105 and HD3086 genotypes (Fig. S1* a, b, c). Among three stress treatments, salinity stress caused 30-40\% decline in seedling lengths and biomass, thus posing much severity for seedling to grow. On the other hand, under water witholding condition, genotypes C 591, C 306, C 518, Drysdale, Gladius, Krl 19, Type 11, PBW 660, PBW 621 and PBW 175 showed a significant (3-10\%) increase in the dry biomass. However, under salt stress condition, genotypes Kharachia, Krl 1-4 and Krl 19 showed a marked increase (5-10\%) in shoot lengths. For instance, ten-fold increase in the dry biomass was observed in Kharchia among all genotypes under salinity stress (Fig S1 a, b, c).

\section{Biochemical parameters}

A significant increase in the accumulation of proline was observed under water withholding condition over water deficit and salt stress in all genotypes (Fig. S2a, b, c). The aver-

*Further details about the Electronic Supplementary Material (ESM) can be found at the end of the article. 
age proline contentwas (2 to 3.4) fold higher in the shoots of genotypes Gladius, Babax, Excalibar, Type 11, C 306, C 518, C591, C 273, Drysdale, PBW 660 and Krichauff under water deficit and water withholding conditions. However, under salinity stress, Kharchia possessed maximum fold increase in proline content followed by Krl 1-4 and Krl 19 ranging from 2 to 2.6 fold (Fig. S2a). On the contrary,wheat genotypes i.e. HD 2967, PBW 621, WH 1105, HD 3086 and PBW 175 possesed lowest proline content when compared to other studied genotypes.

The antioxidant potential of wheat genotypes in terms of percentage scavenging of DPPH was observed in the rangefrom $31.7-85 \%$. A significant increase in the activity was observed in C 306, C 518, Babax, Drysdale and Type 11 genotypes under water deficit and water withholding stress conditions whereas $\mathrm{Krl} \mathrm{1-4}$ and Krl 19 exhibited higher activity under salt conditions. Maximum increase in DPPH radical scavenging activity was observed in the Kharchia under salt stress $(85 \%)$ and water withholding stress $(80 \%)$ conditions (Fig. S2b).

On the flip side, an increase in the TBARS content of wheat seedlings was markedly higher under salt stress condition as compared to water deficit and water withholding condition (Fig. S2c). Among nineteen genotypes, TBARS content was found to be lowest in the shoots of Kharchia(followed by Krl 1-4 and Krl 19 under salinity stress conditionand in C 306, C 273, Gladius, Drysdale and Excalibar under water deficit and water withholding conditions. Whereas, genotypes HD 2967, PBW 621, WH 1105, HD 3086, PBW 660 and PBW 175 exhibited an increase in TBARS content under all studied stress conditions, depicting extensive lipid peroxidation which revealed their salt and drought susceptible nature.

A significant decrease in the contents of $\mathrm{H}_{2} \mathrm{O}_{2}$ was observed in genotypes viz. C 306, C 273, C 591, C 518, Exaclibar, Krichauff, Babax, Drysdale, Gladius, Kharchia, Type 11, Krl 1-4 and Krl 19. While, HD 2967, PBW 621, WH 1105, HD 3086, PBW 660 and PBW 175 genotypes showed a dramatic increase in the contents of shoot $\mathrm{H}_{2} \mathrm{O}_{2}$ under all studied stress conditions (Fig. S2d).

\section{Stress tolerance index and stress resistance level}

Based on stress tolerance index (STI) for the various parameters of shoots (Table S1), the level of stress resistance was calculated for all the nineteen genotypes. The genotypes viz. Excalibar, Krichauff, Babax, Gladius, Krl 1-4 and Krl 19 possessed much higher STI $\geq$ median value for most of the parameters studied i.eproline content, DPPH scavenging activity, length and biomass of shoot or $\leq$ median value for content of $\mathrm{H}_{2} \mathrm{O}_{2}$ and MDA were proposed to have higher stress tolerance toward water deficit, water witholding and salinity conditions. C306 and Kharchia exhibited higher stress tolerance exclusively under water stress and salinity stress respectively. The genotypes viz. HD 2967, PBW 621, WH 1105, HD 3086, PBW 660 and PBW 175 were proposed to be highly susceptible on the basis of lower resistance level. 


\section{Activities of proline metabolizing enzymes}

In the current study, water and salt stress led to upregulation of GDH, P5CS and P5CR activities in shoots of all genotypes which may be a consequence of increased production of proline (Fig. S3a, b, c, d). Higher GDH activity was observed in Gladius (1.91 $\mu \mathrm{mol}$ $\min ^{-1} \mathrm{~g}^{-1} \mathrm{fr} \cdot \mathrm{wt}$ ) under water stress conditions. A similar higher P5CS activity was found in Krichauff was observed $\left(17.2,19.6 \mu \mathrm{mol} \mathrm{min} \mathrm{m}^{-1} \mathrm{~g}^{-1} \mathrm{fr} \cdot \mathrm{wt}\right)$ under water deficit and water withholding condition respectively, further demonstrating its superior tolerance mechanism in terms of maintaining higher proline content. Likewise, higher activity of P5CR in Gladius and Drysdale $\left(1.80 \mu \mathrm{mol} \mathrm{min} \mathrm{m}^{-1} \mathrm{~g}^{-1} \mathrm{fr} \cdot \mathrm{wt}\right)$ further enhanced proline content under both water stress conditions. Interestingly, genotype Kharchia revealed higher GDH, P5CS and P5CR activities under salt stress condition. However, the genotypes PBW 175 and HD 2967 showed a marked decline in the activity of P5CR under salinity stress.

\section{Activity of proline catabolizing enzyme}

Contrary to proline synthesizing enzyme, PDH activity revealed a serious decline in the shoots of studied genotypes (Fig. S3d). Under water deficit and water withholding stress conditions, maximum reduction in the activity of $\mathrm{PDH}$ was observed in Babaxfollowed by Drysdale and Gladius. However, under salinity stress conditions, significant decline in PDH activity was observed in Krl 1-4 (Fig. S3d).

\section{Agglomerative hierarchical clustering and principle component analysis}

Principal component analysis categorized the nineteen genotypes on similarity basis into two major clustures: MC-I; further subdivided into two clusters A and B, and MC-II; possessing C and D sub clusters (Fig. S4). MC-I constituted most of the genotypes having high stress tolerance index and MC-II with genotypes exhibiting low stress tolerance index. The A cluster of MC-I mainly comprised of genotypes i.e. C306, Excalibar, Krichauff, Babax, Drysdale and Gladius that are tolerant towards water scarcity conditions while B cluster chiefly constituted genotypes i.e. Kharchia, Type 11, Krl 1-4 and Krl 19 that more tolerant towards salinity stress. The small subclusters $\mathrm{C}$ of MC-II basically contained the genotypes i.e. C273, C518 and C591 that possesed low stress tolerance index toward water and salt stress conditions. Furthermore, it was the D cluster of MC-II that chiefly constituted the genotypes that exhibit negligible stress tolerance index and were found susceptible towards the stress conditions.

\section{Discussion}

Analysis of growth parametersdepict the inhibition of root/shoot growth and development under water scarcity and salinity stress treatments (Ashraf and Ali 2008). The current study estimated that salt stress treatment decreased the osmotic potential of seedling- 
sto a toxic level as compared to water deficit and water withholding stress conditions, thus finding well correlated earlier reports of Glyn et al. (2011). It may be inferred that the genotypes that exhibited longer shoots with higher biomass viz. Excalibar, C 306, Drysdale, Babax, Gladius, Krichauff under water deficit and water withholding condition and Kharchia, Krl 1-4 and Krl 19 under salt stress condition, might be better responsive and can be correlated with tolerance level towards respective stress conditions. Similar observations on biomass and stress tolerance in wheat genotypes was also reported by Marcińska et al. (2013).

Stress tolerance indices and principal component analysis partitioned all the genotypes under study into four groups i.e drought tolerant (C 306, Excalibar, Krichauff, Babax, Drysdale and Gladius), salt tolerant (Kharchia, Type 11, Krl 1-4 and Krl 19), mid tolerant (C 273, C 518 and C 591) and susceptible (HD 2967, PBW 621, WH 1105, HD 3086, PBW 660 and PBW 175) (Fig. S4). The classification of genotypes was based on the effective relation of proline metabolism and DPPH radical scavenging activity with contents of TBARS and $\mathrm{H}_{2} \mathrm{O}_{2}$ of genotypes under stress conditions. The genotypes exhibiting high tolerance had reduced magnitude of TBARS content in parallel with decreased $\mathrm{H}_{2} \mathrm{O}_{2}$ content and increased proline content and DPPH radical scavenging activity. Higher level of proline and DPPH radical scavenging activity in radicles have been correlated with extreme temperature tolerance (Kang and Saltveit 2002). It was reported that rate of lipid peroxidation (in terms of TBARS content) indicate the sensitivity of plant to stress condition (Jain et al. 2001). The decreased TBARS content indicate reduced oxidative damage to membrane, which thereby attribute towards stress tolerance. The genotypes exhibiting intolerance had high level of TBARS content in their shoots which might result from lack of stress dependent upregulation of antioxidant system as (Kibria et al. 2017). Thus, increased proline content in shoots along with DPPH radical scavenging activity in the tolerant genotypes may be an adaptative strategy to overcome the stress conditions as it supplies energy for growth and lowers the generation of free radicals and reduces the lipid peroxidation linked membrane damage resulting in their stabilization (Abdul-Jaleel et al. 2008; Vetcha et al. 2018).

Enhancement in proline content in tolerant genotypes may be due to the variety of causes.Increased activities of GDH might have played a predominant role in ammonium assimilation and glutamate biosynthesis, leading to an increased glutamate pool for proline synthesis in tolerant genotypes as compared to susceptible genotypes. Increased GDH activity and its significant role in the synthesis of glutamate have been reported in many plant species (Mattioniet al. 1997). As P5CS activity was reported higher than P5CR, so it seems obvious that P5CS activity might be in tight relation to free proline content indicating that glutamate is further converted to proline under stress conditions, and P5CS might be the rate-limiting factor in this pathway. Similar increase in the activity of GDH, P5CS and P5CR was observed in leaves of Cicer arietinum under salt stress (Baher 2015). Proline dehydrogenase converts proline to glutamate. A declining PDH activity was though reported in all studied genotypes under all stress conditions, but the reduction was comparatively higher in tolerant genotypes which might have also influenced the proline accumulation. Among all stress conditions studied, water withholding 
stress induced the stimulation of proline synthesis (via increased GDH, P5CS and P5CR activity) with an enhanced inhibition of oxidation (reduced PDH activity) to a large extent than water deficit and salt stress condition. Therefore, a close correlation between these enzymes and proline content under water withholding stress condition as compared to water deficit and salt stress condition was further confirmed.

The current study stated that all genotypes exhibited marked differences in the physiological growth and proline metabolism under water and salt stress conditions. Among all three stresses,salt stress condition severely limited the growth of all genotypes with severe membrane peroxidation and degradation, due to elevated levels of TBARs and $\mathrm{H}_{2} \mathrm{O}_{2}$. In addition to this, some of the genotypes categoried as tolerant groups i.e drought and salt stress tolerant genotypes possess better membrane stability, antioxidant potential and higher accumulation of proline. Tolerance of these genotypes seems to be related to increased proline content due to over-expression of P5CS and down-regulation of PDH. Data generated from this study will be helpful in carrying out further experimentation in exploring candidate gene behind tolerance in the genotypes, and thus further exploring the potential role of proline under stress conditions.

\section{Acknowledgement}

I acknowledge Dr. Bavita Asthir for her support and help during research work.

\section{References}

AbdElgawad, H., Vos, D.D., Zinta, G., Domagalska, M.A., Beemster, G.T.S., Asard, H. 2015. Grassland species differentially regulate proline concentrations under future climate conditions: an integrated biochemical and modelling approach. New Phytol. 208:354-369.

Abdul-Jaleel, C., Sankar, B., Sridharan, R. Rajaram, P. 2008. Soil salinity alters growth, chlorophyll content, and secondary metabolites accumulation in Catharanthus roseus. Turk. J. Biol. 32:79-83.

Ahmad, P., Hakeem, K.R., Kumar, A., Ashraf, M., Akram, N.A. 2012. Salt-induced changes in photosynthetic activity and oxidative defense system of three cultivars of mustard (Brassica juncea L.). Afr. J. Biotechnol. 11:2694-2703.

Ashraf, M., Ali, Q. 2008. Relative membrane permeability and activities of some antioxidant enzymes as the key determinants of salt tolerance in canola (Brassica napus L.). Environ. Exp. Bot. 63:266-273.

Baher, N. 2015. Agronomic effectiveness of mycorrhizalCicer arietinum L. plants on mechanism of proline in imparting protection, against $\mathrm{NaCl}$ Stress. Adv. Environ. Biol. 9:286-298.

Bhaskara, G.B., Yang, T.H., Verslues, P.E. 2015. Dynamic proline metabolism: importance and regulation in water limited environments. Front. Plant Sci. 6:484.

Cheema, H.S., Singh, B. 1991. Software Statistical Package CPCS-1. Developed at Department of Statistics, Punjab Agricultural University, Ludhiana, India.

Garg, N., Manchanda, G. 2009. ROS generation in plants: Boon or bane? Plant Biosys. 143:81-96.

Glyn, B.A., McKenzie, B.M., Hallett, P.D., Valentine, T.A. 2011. Root elongation, water stress, and mechanical impedance: a review of limiting stresses and beneficial root tip traits. J. Exp. Bot. 62:59-68.

Hameed, A., Hussain, T., Gulzar, S., Aziz, I., Gul, B., Khan, M.A. 2012. Salt tolerance of a cash crop halophyte Suaedafruticosa: biochemical responses to salt and exogenous chemical treatments. Acta Physiol. Planta. 34:2331-2340.

Hussain, M., Mumtaz, S. 2014. Climate change and managing water crisis: Pakistan's perspective. Rev. Environ. Health 29:71-77. 
Jain, M.G., Mathur, S.K., Sarin, N.B. 2001. Ameliorative effects of proline on salt stress induced lipid peroxidation in cell lines of groundnut (Arachis hypogea L.). Plant Cell Rep. 20:463-468.

Kang, H.M., Saltveit, M.E. 2002. Antioxidant enzymes and DPPH radical scavenging activity in chilled and heat shocked rice (Oryza sativa L.) seedling radicles. J. Agric. Food Chem. 50:513-518.

Kaur, N., Kumar, A., Kaur, K., Gupta, A.K., Singh, I. 2014. DPPH radical scavenging activity and contents of $\mathrm{H}_{2} \mathrm{O}_{2}$, melandialdehyde and proline in determining salinity tolerance in chickpea seedlings. Int. J. Biochem. Biophys. 51:407-415.

Kavi-Kishor, P.B., Sangam, S., Amrutha, R.N., Sri-Laxmi, P., Naidu, K.R., Rao, K.R.S.S., Rao, S., Reddy, K.J., Theriappan, P., Sreenivasulu, N. 2005. Regulation of proline biosynthesis, degradation, uptake and transport in higher plants: Its implications in plant growth and abiotic stress tolerance. Curr. Sci. 88:3.

Kibria, M.G., Hossain, M., Murata, Y., Hoque, M.A. 2017. Antioxidant defense mechanisms of salinity tolerance in rice genotypes. Rice Sci. 24:155-162.

Li, J.T., Qiu, Z.B., Zhang, X.W., Wang, L.S. 2011. Exogenous hydrogen peroxide can enhance tolerance of wheat seedlings to salt stress. Acta Physiol. Plant. 33:835-842.

Lobell, D.B., Schlenker, W., Costa-Roberts, J. 2011. Climate trends and global crop production since 1980. Sci. 333:616-620.

Mahboob, W., Khan, M.A., Shirazi, M.U., Mumtaz, S., Shereen, A. 2018. Using growth and ionic contents of wheat seedlings as rapid screening tool for salt tolerance. J. Crop Sci. Biotech. 21:173-181.

Marcińska, I., Czyczyło-Mysza, I., Skrzypek, E., Filek, M., Grzesiak, S., Grzesiak, M.T., Janowiak, F., Hura, T., Dziurka, M., Dziurka, K., Nowakowska, A., Steve, A. 2013. Quarrie Impact of osmotic stress on physiological and biochemical characteristics in drought-susceptible and drought-resistant wheat genotypes. Acta Physiol. Plant 35:451-461.

Mattioni, C., Gabbrielli, R., Vangronsveld, J., Clijsters, H. 1997. Nickel and cadmium toxicity and enzymatic activity in Ni-tolerant and non-tolerant populations of Sileneitalica. Pers. J. Plant Physiol. 150:173-177.

Miller, G., Honig, A., Stein, H., Suzuki, N., Mittler, R., Zilberstein, A. 2009. Unraveling delta1-pyrroline5-carboxylate proline cycle in plants by uncoupled expression of proline oxidation enzymes. J. Biol. Chem. 284:26482-26492.

Mwadzingeni, L., Shimelis, H., Tesfay, S., Tsilo, T.J. 2016. Screening of bread wheat genotypes for drought tolerance using phenotypic and proline analyses. Front. Plant Sci. 7:1276.

Rao, A., Ahmad, S.D., Sabir, S.M., Awan, S.I., Hameed, A., Abbas, S.R., Shehzad, M., Khan, M.F., Shafique, S., Ahmad, Z. 2013. Detection of saline tolerant wheat cultivars (Triticumaestivum L.) using lipid peroxidation, antioxidant defense system, glycinebetaineand proline contents. J. Animal Plant Sci. 23:1742-1748.

Sahitya, U.L., Krishna, M., Deepthi, R.S., Prasad, G.S., Kasim, D.P. 2018. Seed antioxidants interplay with drought stress tolerance indices in chilli (Capsicum annuum L.) seedlings. BioMed res. international. 2018: 1605096.

Szabados, L., Savouré, A. 2009. Proline: a multifunctional amino acid. Trends Plant Sci. 15:1360-1385.

Vetcha, A., Pidakala, R., Bapatla, R.B., Booba, S., Agepati, S.R. 2018. Oxidative stress induced in chloroplast or mitochondria promotes proline accumulation in leaves of pea (Piscum sativum): another example of chloroplast-mitochondria interactions. Protoplasma. 2018:1-9.

Yu, G.H., Zhang, X., Ma, H.X. 2015. Changes in the physiological parameters of SbPIP1-transformed wheat plants under salt stress. International J genomics. 2015:384356. doi:10.1155/2015/384356.

\section{Electronic Supplementary Material (ESM)}

Electronic Supplementary Material (ESM) associated with this article can be found at the website of CRC at http://www.akademiai.com/content/120427/

Electronic Supplementary Table S1. Content of TBARS under water and salt stress condition in nineteen wheat genotypes 
Electronic Supplementary Figure S1. Effect of water deficit, water withholding and salinity stress on the wheat genotypes in shoot physiological parameters. (a) Shoot length $(\mathrm{G}=0.863289, \mathrm{~T}=0.396104, \mathrm{G} \times \mathrm{T}=1.72658)$ (b) Fresh weight $(\mathrm{G}=0.00503471, \mathrm{~T}=0.00231008 \mathrm{E}, \mathrm{G} \times \mathrm{T}=0.0100694)$ (c) Dry weight $(\mathrm{G}=0.0019694$, $\mathrm{T}=0.000903622, \mathrm{G} \times \mathrm{T}=0.00393880$ ) where $\mathrm{G}$ is genotypes, $\mathrm{T}$ is treatment and $\mathrm{G} \times \mathrm{T}$ is genotype and treatment interaction. The values of $\mathrm{G}, \mathrm{T}$ and $\mathrm{G} \times \mathrm{T}$ are the critical differences at $5 \%$ level of difference while vertical bars on graph represent standard errors.

Electronic Supplementary Figure S2. Effect of water deficit, water withholding and salinity stress on the proline content of wheat genotypes. (a) Proline $(\mathrm{G}=0.405731, \mathrm{~T}=0.186162, \mathrm{G} \times \mathrm{T}=0.811462)$, (b) $\mathrm{DPPH}$ $(\mathrm{G}=6.91965, \mathrm{~T}=\mathrm{NS}, \mathrm{G} \times \mathrm{T}=13.8393),(\mathrm{c}) \mathrm{TBARS}(\mathrm{G}=0.191432, \mathrm{~T}=0.0878349, \mathrm{G} \times \mathrm{T}=0.382864)$ and (d) $\mathrm{H}_{2} \mathrm{O}_{2}(\mathrm{G}=0.0119863, \mathrm{~T}=0.00549970, \mathrm{G} \times \mathrm{T}=0.0239727)$ where $\mathrm{G}$ is genotypes, $\mathrm{T}$ is treatment and $\mathrm{G} \times \mathrm{T}$ is genotype and treatment interaction. The values of $\mathrm{G}, \mathrm{T}$ and $\mathrm{G} \times \mathrm{T}$ are the critical differences at $5 \%$ level of difference while vertical bars on graph represent standard errors.

Electronic Supplementary Figure S3. Effect of water deficit, water withholding and salinity stress on the activities of proline metabolizing enzymes of wheat genotypes. (a) $\mathrm{GDH}(\mathrm{G}=0.0713407, \mathrm{~T}=0.0327334$, $\mathrm{G} \times \mathrm{T}=0.142681)(\mathrm{b}) \mathrm{P} 5 \mathrm{CS}(\mathrm{G}=1.32324, \mathrm{~T}=\mathrm{NS}, \mathrm{G} \times \mathrm{T}=2.64647)(\mathrm{c}) \mathrm{P} 5 \mathrm{CR}(\mathrm{G}=0.0624154, \mathrm{~T}=0.0286382$, $\mathrm{G} \times \mathrm{T}=0.124831)(\mathrm{d}) \mathrm{PDH}(\mathrm{G}=0.0176580, \mathrm{~T}=\mathrm{NS}, \mathrm{G} \times \mathrm{T}=0.0353160)$ where $\mathrm{G}$ is genotypes, $\mathrm{T}$ is treatment and $\mathrm{G} \times \mathrm{T}$ is genotype and treatment interaction. The values of $\mathrm{G}, \mathrm{T}$ and $\mathrm{G} \times \mathrm{T}$ are the critical differences at $5 \%$ level of difference while vertical bars on graph represent standard errors.

Electronic Supplementary Figure S4. Hierarchical agglomerative clusturing 